\title{
Patient surveillance after treatment for soft-tissue sarcoma
}

\author{
FRANK E. JOHNSON ${ }^{1,2}$, KEITA SAKATA ${ }^{2}$, SUCHIRA SARKAR ${ }^{3}$, RICCARDO A. AUDISIO ${ }^{3}$, \\ WILLIAM G. KRAYBILL ${ }^{4}$, JOHN F. GIBBS ${ }^{4}$, ALAN L. BEITLER ${ }^{4}$ and KATHERINE S. VIRGO ${ }^{1,2}$ \\ ${ }^{1}$ Department of Surgery, Saint Louis University Medical Center, 3635 Vista Avenue, P.O. Box 15250, St. Louis, \\ MO 63110-0250; ${ }^{2}$ Surgical Service, Department of Veterans Affairs Medical Center, 915 North Grand Blvd., \\ Saint Louis, MO 63106, USA; ${ }^{3}$ University of Liverpool, Department of Surgery, St. Helens \\ and Knowsley University Hospitals NHS Trust, Liverpool, UK; ${ }^{4}$ Department of Surgery, Roswell Park \\ Cancer Institute, Roswell Park Cancer Institute, Elm \& Carlton Streets, Buffalo, NY 14263, USA
}

Received June 24, 2010; Accepted September 6, 2010

DOI: 10.3892/ijo_00000843

\begin{abstract}
About $1 \%$ of all cancers are soft tissue sarcomas (STS); about $60 \%$ of these occur in the extremities. Posttreatment surveillance programs are designed to identify recurrence, new primary cancers, and complications of therapy early enough to increase survival duration and quality of life. The intensity of surveillance varies among surgeons. We hypothesized that geographic factors would account for much of this variation. The 1,592 members of the Society of Surgical Oncology were surveyed regarding their personal postoperative STS surveillance strategy using standardized clinical vignettes and a questionnaire based on the vignettes. Practice patterns were analyzed by US Census Region, Metropolitan Statistical Area (MSA), and managed care organization (MCO) penetration rate, using repeated measures analysis of variance. The study end-point was surveillance intensity. Mean follow-up intensity for the 12 surveillance modalities on the questionnaire was highly correlated with tumor size, grade, and year post surgery. Controlling for tumor stage, grade, and year post surgery, the practice location of the surgeon infrequently impacted surveillance intensity. MSA was a significant $(\mathrm{p}<0.05)$ predictor only of office visit frequency. MCO penetration rate significantly predicted only the frequency of urinalysis and tumor-site MRI. US Census Region significantly predicted only the frequency of LFTs. Geographic factors do not generally predict self-reported surveillance practice patterns for patients after curative-intent STS surgery. The overall variation in follow-up intensity appears to reflect factors not evaluated, such as the absence of high-quality evidence supporting any particular strategy and the quality of patients' insurance.
\end{abstract}

Correspondence to: Dr Frank E. Johnson, Department of Surgery, Saint Louis University Medical Center, 3635 Vista Ave. at Grand Blvd., P.O. Box 15250, St. Louis, MO 63110-0250, USA

E-mail: frank.johnson1@va.gov

Key words: current practice patterns, geographic variation, surveillance, sarcoma

\section{Introduction}

Only $1 \%$ of all newly diagnosed cancers are soft tissue sarcomas (STS), of which 59\% originate within an extremity. Following potentially curative treatment, one-third of patients suffer recurrence, typically within two years (1-4). However, recurrence after five years is not unknown (1,5-7). It is therefore essential that a follow-up program be implemented to reassure patients of their disease-free status or to identify recurrences or new primaries $(1,7,8)$. Various groups, such as the National Comprehensive Cancer Network (NCCN), have recommended various follow-up protocols for STS. However, there remains a lack of compliance with these recommendations and follow-up intensity varies worldwide (8-10). Geographical factors could account for variation in posttreatment surveillance. Such variation would suggest that services are either overutilized or underutilized (11-13).

Past research performed to analyze the effect of geographic location on follow-up strategies utilized have used stratification by metropolitan statistical area (MSA), state, county and US Census Region (11-13). MSAs are areas with adequate population to be considered primary census areas by the US Bureau of the Census as defined by the Office of Management and Budget (14). Comparisons of MSAs and small towns and rural areas allow differences in test utilization by geographical locations to be identified (14-17).

Enrollment into managed care organizations (MCOs) has been increasing steadily since the 1980s. MCOs use numerous payment and management mechanisms to control costs, which include revenues flowing to healthcare providers, such as hospitals $(18,19)$. Thus, the greater the penetration rate of MCOs in particular geographical locations, the more likely hospital revenues and profits are to be constrained. As a result, hospitals then have more incentive to control costs. This has raised much debate over the quality of care MCOs provide.

Therefore, it is reasonable to question whether physicians in areas with higher managed-care enrollment rates use fewer tests during follow-up than physicians in areas with low enrollment rates.

A survey was conducted recently to identify the surveillance strategies chosen by members of the Society of Surgical 
Oncology (SSO) in the management of patients with extremity soft tissue sarcoma following potentially curative treatment (20). Results obtained from the survey showed that intensity of surveillance following primary treatment varied immensely. Previous analyses of the study data have tested whether the variation in utilization of tests is linked to tumor size and grade or to physician age $(21,22)$. The focus of the current analysis was to assess the influence of geographical location (MSA and US Census Region) and MCO penetration rate on the utilization of follow-up modalities.

\section{Materials and methods}

All 1,592 members of the SSO were surveyed regarding their follow-up strategies for patients with extremity STS after potentially curative treatment. The participants were mailed a cover letter outlining the purpose of the study along with a six-page survey and a self-addressed, stamped return envelope. Details of the methods and main results have already been published (20). Each member was asked to report the frequency of his or her personal follow-up evaluation for each of 12 follow-up modalities. Vignettes describing a low-grade small tumor ( $\leq 5$ in diameter), a low-grade large tumor $(>5 \mathrm{~cm}$ in diameter), a high-grade small tumor and a high-grade large tumor during years 1-5 and year 10 posttreatment were created.

The modalities used in the survey were office visit, complete blood count, liver function tests, serum electrolyte levels, urinalysis, erythrocyte sedimentation rate, chest X-ray, chest CT, X-ray of the tumor site, CT of the tumor site, MRI of the tumor site, and bone scan. One of the modalities was categorized as 'other' in case a physician used a test which was not noted in the survey. Because many advanced statistical methods require independence of observations, correlation analysis was used to determine if an individual's response for year 1 for a particular modality was independent of the response given for years 2-5 and 10 for that same modality. Similarly, correlation analysis was used to determine if an individual's response for a particular follow-up modality for a patient with a low-grade small tumor was independent of the response given for that modality for a patient with a low-grade large tumor.

Survey responses were grouped and analyzed by the MSA, US Census Region and MCO penetration rate corresponding to the area from which each reporting physician had responded. Because the number of MSAs is large and the number of respondents per MSA was often small, each MSA with fewer than 15 respondents was placed in the category 'other MSA'. All respondents from non-MSAs (small towns and rural areas) were grouped into a separate category. Non-US respondents were labeled as foreign and placed in a separate group.

To broaden the analysis to one based on larger geographic regions, survey responses were then regrouped based on the U.S. Census Regions. The standard Census Regions were modified slightly to include Puerto Rico in the South Atlantic Region. In addition, the results obtained from surgeons in the Mountain and Pacific Regions were combined due to the small number of respondents from each Region. This combined Region was categorized as Pacific Region. A tenth Region was created to include responses from SSO members from outside the US.
Lastly, MCO penetration data for cities and states (obtained from the National Research Corp.) were added to the database (23). Based on a frequency analysis of MCO penetration rates by state, a categorical variable was created that corresponded to $0.00-29.3,29.4-37.7,38.3-42.2$ and $44.9-57.1 \%$ enrollment. Repeated-measures analysis of variance was used to compare practice patterns by MSA, US Census Region and MCO penetration rates across STS grade, size and postoperative years. Statistical significance was set at $\mathrm{p}<0.05$.

\section{Results}

Of the 1,592 SSO members who received the survey, 714 (45\%) responded. A total of 343 of the 714 (48\%) performed sarcoma surgery; 318 of these $343(93 \%)$ conducted surgery and provided long-term follow-up, which equates to $44 \%$ of respondents (318/714) who provided the evaluable data reported below. The demographic profile of the respondents has already been published (20).

Correlation analysis revealed that mean follow-up intensity for the majority of modalities was highly correlated across tumor grade, size, and year post surgery. This means that the strategy used for follow-up by an individual respondent in year 1 was highly correlated with the strategy selected for year 2, which was also highly correlated with the strategy selected for year 3, and so on. Similarly, the strategy implemented by given respondent for follow-up of a patient with a lesion of particular size and grade was highly correlated across the entire follow-up period and was also highly correlated with the responses for lesions of other sizes and grades. In addition, the modalities used by a specific surgeon for surveillance of a patient with a sarcoma of a particular size and grade were highly correlated across all post surgery years with the modalities chosen by that surgeon for sarcomas of other sizes and grades.

Six MSAs had 15 or more respondents. Table I lists the means and standard deviations for each of the commonly used modalities by MSA for a high-grade STS $>5 \mathrm{~cm}$ in diameter and year 1 post surgery. Commonly employed modalities were office visit, chest X-ray, complete blood count and liver function tests. Surveillance varied only modestly among grades, sizes, and years, so only data for this particular vignette and year 1 post surgery is shown. Buffalo-Niagara Falls and New York City were consistently the MSAs with the highest frequency of utilization of the seven most common modalities across grades, sizes, and post surgery years. Non-MSA regions also recorded a high frequency of use for the common modalities. The Houston and Los Angeles MSAs had consistently low-frequency users of the commonest modalities. Although the Philadelphia MSA respondents used office visits infrequently, they used the other modalities frequently. For the uncommon modalities (not shown on the table), very small means and standard deviations were observed.

Significant main effects $(\mathrm{p}<0.05)$ were obtained for grade, size, and year for all modalities except electrolytes, urinalysis, erythrocyte sedimentation rate, site X-ray, site CT and bone scan. The effect of MSA was only significant for office visit, urinalysis, erythrocyte sedimentation rate, site X-ray and site CT. A number of two-way interactions had significant main 
Table I. Frequency of test utilization analyzed by MSA in postoperative year 1 after surgery for high-grade (TNM Stage II-III) soft tissue sarcoma of the extremity measuring $>5 \mathrm{~cm}(\mathrm{n}=282){ }^{\mathrm{a}}$

\begin{tabular}{|c|c|c|c|c|c|c|}
\hline \multirow[b]{2}{*}{ MSA } & \multicolumn{6}{|c|}{ Modality (mean \pm standard deviation) } \\
\hline & Office visit ${ }^{\mathrm{b}}$ & $\mathrm{CBC}$ & LFTs & Electrolytes & UA & ESR \\
\hline Buffalo, Niagara Falls & $4.3 \pm 0.8$ & $2.1 \pm 1.7$ & $2.1 \pm 1.7$ & $0.8 \pm 1.4$ & $0.1 \pm 0.4$ & $0.0 \pm 0.0$ \\
\hline Non-MSA ${ }^{c}$ & $3.9 \pm 1.3$ & $1.2 \pm 1.8$ & $1.4 \pm 1.8$ & $0.3 \pm 0.9$ & $0.1 \pm 0.6$ & $0.0 \pm 0.0$ \\
\hline New York City & $3.8 \pm 0.9$ & $0.7 \pm 1.1$ & $0.7 \pm 1.1$ & $0.1 \pm 0.2$ & $0.2 \pm 0.5$ & $0.0 \pm 0.0$ \\
\hline Other $\mathrm{MSA}^{\mathrm{d}}$ & $3.8 \pm 1.0$ & $1.0 \pm 1.4$ & $1.2 \pm 1.4$ & $0.4 \pm 1.1$ & $0.1 \pm 0.6$ & $0.0 \pm 0.3$ \\
\hline Chicago & $3.6 \pm 0.7$ & $0.7 \pm 1.2$ & $0.9 \pm 1.3$ & $0.6 \pm 1.4$ & $0.0 \pm 0.0$ & $0.0 \pm 0.0$ \\
\hline Los Angeles & $3.6 \pm 1.0$ & $0.5 \pm 1.0$ & $0.8 \pm 1.1$ & $0.4 \pm 1.0$ & $0.1 \pm 0.3$ & $0.1 \pm 0.3$ \\
\hline Philadelphia & $3.4 \pm 0.9$ & $1.3 \pm 1.4$ & $1.8 \pm 1.6$ & $0.1 \pm 0.3$ & $0.3 \pm 0.5$ & $0.1 \pm 0.4$ \\
\hline Houston & $3.3 \pm 1.2$ & $1.3 \pm 0.6$ & $1.3 \pm 0.6$ & $0.0 \pm 0.0$ & $0.0 \pm 0.0$ & $0.0 \pm 0.0$ \\
\hline Total & $3.8 \pm 1.0$ & $1.0 \pm 1.4$ & $1.2 \pm 1.4$ & $0.4 \pm 1.0$ & $0.1 \pm 0.6$ & $0.0 \pm 0.3$ \\
\hline
\end{tabular}

Modality (mean \pm standard deviation) high grade $>5 \mathrm{~cm}$

\begin{tabular}{|c|c|c|c|c|c|c|}
\hline \multirow[b]{2}{*}{ MSA } & \multirow{2}{*}{ CXR } & \\
\hline & & Chest CT & Site X-ray & Site CT & Site MRI & Bone scan \\
\hline Buffalo, Niagara Falls & $2.0 \pm 1.3$ & $0.1 \pm 0.4$ & $0.0 \pm 0.0$ & $0.1 \pm 0.4$ & $0.1 \pm 0.4$ & $0.0 \pm 0.0$ \\
\hline Non-MSA ${ }^{c}$ & $1.7 \pm 1.5$ & $1.0 \pm 1.3$ & $0.4 \pm 1.0$ & $0.8 \pm 1.3$ & $0.7 \pm 1.1$ & $0.1 \pm 0.2$ \\
\hline New York City & $1.9 \pm 1.5$ & $0.8 \pm 1.1$ & $0.2 \pm 0.6$ & $0.5 \pm 0.8$ & $0.3 \pm 0.6$ & $0.1 \pm 0.5$ \\
\hline Other MSA ${ }^{d}$ & $1.9 \pm 1.1$ & $0.7 \pm 1.0$ & $0.1 \pm 0.4$ & $0.4 \pm 0.8$ & $0.6 \pm 0.9$ & $0.1 \pm 0.4$ \\
\hline Chicago & $1.6 \pm 1.4$ & $1.0 \pm 1.3$ & $0.1 \pm 0.5$ & $0.6 \pm 0.8$ & $0.5 \pm 0.7$ & $0.1 \pm 0.3$ \\
\hline Los Angeles & $1.9 \pm 1.1$ & $0.6 \pm 1.1$ & $0.3 \pm 1.0$ & $0.4 \pm 0.7$ & $0.5 \pm 1.1$ & $0.0 \pm 0.0$ \\
\hline Philadelphia & $1.4 \pm 0.7$ & $0.4 \pm 0.7$ & $0.0 \pm 0.0$ & $0.6 \pm 0.9$ & $1.3 \pm 0.9$ & $0.1 \pm 0.4$ \\
\hline Houston & $1.7 \pm 0.6$ & $0.0 \pm 0.0$ & $0.0 \pm 0.0$ & $0.0 \pm 0.0$ & $0.7 \pm 0.6$ & $0.0 \pm 0.0$ \\
\hline Total & $1.8 \pm 1.2$ & $0.7 \pm 1.0$ & $0.1 \pm 0.6$ & $0.4 \pm 0.9$ & $0.6 \pm 0.9$ & $0.1 \pm 0.4$ \\
\hline
\end{tabular}

MSA, metropolitan statistical area; TNM, tumor, node, metastasis; CBC, complete blood count; LFTs, liver function tests; UA, urinalysis; ESR, erythrocyte sedimentation rate; CXR, chest X-ray; CT, computed tomography; MRI, magnetic resonance imaging. The value in each cell represents the number of times the practitioner requests each test or other modality. ${ }^{\mathrm{a}}$ Restricted to U.S. respondents only due to lack of MSA data for foreign respondents. ${ }^{\mathrm{b}} \mathrm{p}<0.05$. Significant differences exist among the cells in the column. ${ }^{\mathrm{c}}$ Rural areas and small towns with no MSA code. ${ }^{\mathrm{d}} \mathrm{MSAs}$ with $<15$ responses.

effects. The interactions of grade and MSA was significant for urinalysis and electrolytes. The size and year interaction was significant for chest X-ray and site MRI. There were significant grade and year main effects for office visit, liver function tests, chest X-ray and chest CT. Finally, the two-way interactions of grade and size was significant for liver function tests and bone scan. Three-way interactions of size, year and MSA were significant for urinalysis, site MRI and bone scan; the interaction of grade, year and MSA for erythrocyte sedimentation rate and other tests and the interactions of grade, size, and MSA for bone scan were also significant.

The mean and standard deviation for all modalities by MCO penetration rates for large high-grade STS in post surgery year 1 are listed in Table II. Respondents from states with MCO penetration rates of 29.4-37.7 and 44.9-57.1\% had the highest frequency of usage for the common modalities, such as office visit, CBC and chest X-ray. Surgeons from states with $\mathrm{MCO}$ penetration rates of 38.3-42.2\% reported the highest usage for uncommon modalities.
This analysis only disclosed significant main effects of MCO penetration rate for office visit, urinalysis and site MRI. The grade, size, and year main effects were significant for all modalities with the exception of electrolytes, urinalysis, erythrocyte sedimentation rate, site X-ray, site MRI and 'other' tests. The analysis also found two-way and three-way interactions but no four-way interactions. The interaction of grade and year was significant for the common modalities. The interaction of size and year and the interaction of grade and size were only significant for modalities that were used infrequently. The interaction of year and MCO penetration rate was only significant for urinalysis and bone can. Two three-way interactions were significant. Grade, size and year were significant for electrolytes and site CT scan while the interaction of grade, size and MCO penetration rate was significant for site MRI only.

Table III lists the mean and standard deviation for each of the commonly used follow-up modalities by US Census Region for large high-grade STS and year 1 post surgery. The Mid- 
Table II. Frequency of test utilization analyzed by Managed Care Organization (MCO) penetration rate in postoperative year 1 after surgery for high-grade (TNM Stage II-III) soft tissue sarcoma of the extremity measuring $>5 \mathrm{~cm}(\mathrm{n}=282)^{{ }^{a}}$

Modality

\section{MCO}

penetration

rates $(\%)$

$$
\text { Office visit }
$$

CBC

LFTs

Electrolytes

$\mathrm{UA}^{\mathrm{b}}$

ESR

\begin{tabular}{lllllll}
\hline $0.00-29.3$ & $3.8 \pm 1.0$ & $0.8 \pm 1.2$ & $1.0 \pm 1.3$ & $0.3 \pm 0.8$ & $0.0 \pm 0.1$ & $0.0 \pm 0.1$ \\
$29.4-37.7$ & $3.8 \pm 1.0$ & $1.2 \pm 1.6$ & $1.3 \pm 1.6$ & $0.4 \pm 1.1$ & $0.1 \pm 0.4$ & $0.1 \pm 0.5$ \\
$38.3-42.2$ & $3.5 \pm 1.1$ & $1.0 \pm 1.3$ & $1.3 \pm 1.4$ & $0.4 \pm 1.0$ & $0.3 \pm 0.8$ & $0.0 \pm 0.2$ \\
$44.9-57.1$ & $3.8 \pm 1.0$ & $1.0 \pm 1.5$ & $1.1 \pm 1.5$ & $0.5 \pm 1.2$ & $0.2 \pm 0.8$ & $0.1 \pm 0.5$ \\
Total & $3.6 \pm 1.0$ & $1.0 \pm 1.4$ & $1.2 \pm 1.4$ & $0.4 \pm 1.0$ & $0.1 \pm 1.0$ & $0.1 \pm 0.4$ \\
\hline
\end{tabular}

Modality

MCO

penetration

rates $(\%)$

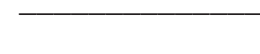

\begin{tabular}{lcccccc} 
rates $(\%)$ & CXR & Chest CT & Site X-ray & Site CT & Site MRI $^{\text {b }}$ & Bone scan \\
\hline $0.00-29.3$ & $1.8 \pm 1.1$ & $0.5 \pm 0.9$ & $0.1 \pm 0.6$ & $0.3 \pm 0.7$ & $0.5 \pm 0.8$ & $0.0 \pm 0.2$ \\
$29.4-37.7$ & $1.9 \pm 1.1$ & $0.7 \pm 1.1$ & $0.1 \pm 0.3$ & $0.5 \pm 1.0$ & $0.6 \pm 0.9$ & $0.1 \pm 0.6$ \\
$38.3-42.2$ & $1.7 \pm 1.1$ & $1.0 \pm 1.2$ & $0.2 \pm 0.7$ & $0.6 \pm 0.9$ & $0.9 \pm 1.0$ & $0.1 \pm 0.4$ \\
$44.9-57.1$ & $1.9 \pm 1.4$ & $0.7 \pm 1.0$ & $0.2 \pm 0.9$ & $0.4 \pm 0.7$ & $0.6 \pm 0.9$ & $0.1 \pm 0.3$ \\
Total & $1.8 \pm 1.2$ & $0.7 \pm 1.0$ & $0.1 \pm 0.6$ & $0.4 \pm 0.8$ & $0.6 \pm 0.9$ & $0.1 \pm 0.4$ \\
\hline
\end{tabular}

TNM, tumor, node, metastasis; CBC, complete blood count; LFTs, liver function tests; UA, urinalysis; ESR, erythrocyte sedimentation rate; CXR, chest X-ray; CT, computed tomography; MRI, magnetic resonance imaging. The value in each cell represents the number of times the practitioner requests each test or other modality. ${ }^{a}$ Restricted to US respondents only due to non-applicability of the MCO penetration rate concept to foreign responses. ${ }^{b} \mathrm{p}<0.05$. Significant differences exist among the cells in the column.

Atlantic Region generally recorded the highest frequency of use for many of the modalities used although West South Central Region respondents also recorded a high frequency of utilization. Respondents from the New England and East South Central Regions reported the least frequent use of the common modalities.

US Census Region had a significant effect only for liver function tests and chest X-ray. There were also significant main effects of tumor grade, posttreatment year and tumor size for all modalities. There were few significant two-way and three-way interactions. The interaction of grade and year was significant for all modalities with the exception of electrolytes, urinalysis, site X-ray and 'other' tests. The twoway interaction of year and size was significant for $\mathrm{CBC}$, chest X-ray, site CT and site MRI. Posttreatment year and Census Region had significant main effects on chest X-ray also. Few three-way interactions were identified. The threeway interaction of grade, year and Region had a significant main effect for site CT only, while the interaction of grade, size and year were significant for CBC.

\section{Discussion}

STSs are uncommon and affect about 5,000-6,000 people in the US each year. Approximately $50 \%$ of these occur in the extremities $(1,6,9)$. Despite optimal treatment of primary tumors, about a third of patients with extremity tumors experience local or distant recurrence at a median diseasefree interval of 18 months $(1,9,13)$. Various studies have shown that these recurrences can be treated with good results, providing a compelling rationale for surveillance testing. The purposes of such regimens include providing reassurance to patients that no recurrence has occurred and identifying recurrence with the premise that early recognition and treatment can prolong survival (1). The follow-up procedures now utilized in routine clinical practice or in research protocols have been developed without stringent clinical studies. Nonetheless, advisory guidelines have been produced by the National Comprehensive Cancer Network (NCCN) for routine surveillance testing following curative-intent treatment, but compliance with these guidelines is poor, as our data confirm. One reason for this variation may be the geographical locations of the practicing physicians. Health care services vary in cost, quality, accessibility, and other dimensions across the world and within countries and are frequently changing. It appears that there are differences in practices among physicians as well. They are due to such factors as availability of resources, guidelines for their applications, differences in training of physicians, and variation in patient expectations. 
Table III. Frequency of test utilization analyzed by US Census Region in postoperative year 1 after surgery for high-grade (TNM Stage II-III) soft tissue sarcoma of the extremity measuring $>5 \mathrm{~cm}(\mathrm{n}=318)$.

Modality (mean \pm standard deviation)

\begin{tabular}{|c|c|c|c|c|c|c|}
\hline \multirow[b]{2}{*}{ MSA } & \\
\hline & Office visit & $\mathrm{CBC}$ & $\mathrm{LFTs}^{\mathrm{a}}$ & Electrolytes & UA & ESR \\
\hline West North Central & $3.9 \pm 1.4$ & $0.8 \pm 1.1$ & $1.0 \pm 1.4$ & $0.4 \pm 0.9$ & $0.3 \pm 0.9$ & $0.1 \pm 0.3$ \\
\hline Pacific & $3.4 \pm 1.0$ & $0.8 \pm 1.3$ & $1.1 \pm 1.2$ & $0.4 \pm 1.1$ & $0.1 \pm 0.4$ & $0.0 \pm 0.2$ \\
\hline Mid Atlantic & $3.8 \pm 1.0$ & $1.2 \pm 1.5$ & $1.4 \pm 1.6$ & $0.4 \pm 0.9$ & $1.2 \pm 0.6$ & $0.0 \pm 0.2$ \\
\hline West South Central & $3.8 \pm 1.0$ & $1.4 \pm 1.7$ & $1.4 \pm 1.7$ & $0.5 \pm 1.4$ & $0.0 \pm 0.0$ & $0.2 \pm 0.9$ \\
\hline East North Central & $3.8 \pm 0.9$ & $0.8 \pm 1.3$ & $1.1 \pm 1.3$ & $0.3 \pm 1.0$ & $0.0 \pm 0.0$ & $0.0 \pm 0.0$ \\
\hline South Atlantic ${ }^{b}$ & $3.7 \pm 1.2$ & $1.0 \pm 1.4$ & $1.2 \pm 1.4$ & $0.4 \pm 0.9$ & $1.2 \pm 0.6$ & $0.0 \pm 0.1$ \\
\hline East South Central & $3.7 \pm 1.1$ & $0.6 \pm 1.1$ & $0.6 \pm 1.1$ & $0.3 \pm 0.6$ & $0.1 \pm 0.2$ & $0.0 \pm 0.0$ \\
\hline Foreign & $3.6 \pm 0.9$ & $0.8 \pm 1.3$ & $0.8 \pm 1.2$ & $0.4 \pm 1.1$ & $0.2 \pm 0.5$ & $0.1 \pm 0.4$ \\
\hline New England & $3.5 \pm 0.8$ & $0.4 \pm 1.1$ & $0.5 \pm 1.1$ & $0.3 \pm 1.0$ & $0.1 \pm 0.2$ & $0.0 \pm 0.0$ \\
\hline Total & $3.8 \pm 1.0$ & $0.9 \pm 1.4$ & $1.1 \pm 1.4$ & $0.4 \pm 1.0$ & $0.1 \pm 0.5$ & $0.0 \pm 0.3$ \\
\hline
\end{tabular}

Modality (mean \pm standard deviation)

\begin{tabular}{|c|c|c|c|c|c|c|}
\hline Region & CXR & Chest CT & Site X-ray & Site CT & Site MRI & Bone scan \\
\hline West North Central & $2.3 \pm 1.2$ & $1.0 \pm 1.4$ & $0.3 \pm 1.2$ & $0.5 \pm 1.2$ & $0.8 \pm 1.4$ & $0.3 \pm 0.5$ \\
\hline Pacific & $1.8 \pm 1.2$ & $0.6 \pm 0.9$ & $0.2 \pm 0.7$ & $0.3 \pm 0.6$ & $0.6 \pm 0.9$ & $0.1 \pm 0.2$ \\
\hline Mid Atlantic & $1.9 \pm 1.3$ & $0.6 \pm 1.0$ & $0.2 \pm 0.8$ & $0.5 \pm 0.9$ & $0.4 \pm 0.7$ & $0.1 \pm 0.3$ \\
\hline West South Central & $1.9 \pm 1.2$ & $0.7 \pm 1.3$ & $0.0 \pm 0.0$ & $0.4 \pm 0.9$ & $0.7 \pm 1.0$ & $0.1 \pm 0.2$ \\
\hline East North Central & $1.8 \pm 1.2$ & $0.7 \pm 1.1$ & $0.1 \pm 0.3$ & $0.5 \pm 0.8$ & $0.5 \pm 0.7$ & $0.0 \pm 0.2$ \\
\hline South Atlantic ${ }^{b}$ & $1.8 \pm 1.2$ & $0.7 \pm 1.1$ & $0.2 \pm 0.7$ & $0.4 \pm 0.8$ & $0.7 \pm 0.9$ & $0.0 \pm 0.2$ \\
\hline East South Central & $1.7 \pm 0.8$ & $0.4 \pm 1.0$ & $0.1 \pm 0.3$ & $0.5 \pm 1.0$ & $0.4 \pm 1.0$ & $0.2 \pm 0.9$ \\
\hline Foreign & $2.4 \pm 1.4$ & $0.6 \pm 0.9$ & $0.1 \pm 0.4$ & $0.8 \pm 1.0$ & $0.7 \pm 0.9$ & $0.1 \pm 0.4$ \\
\hline New England & $1.7 \pm 1.2$ & $0.9 \pm 1.2$ & $0.1 \pm 0.5$ & $0.4 \pm 0.7$ & $0.8 \pm 0.9$ & $0.1 \pm 0.5$ \\
\hline Total & $1.9 \pm 1.2$ & $0.7 \pm 1.0$ & $0.1 \pm 0.6$ & $0.5 \pm 0.8$ & $0.6 \pm 0.9$ & $0.1 \pm 0.4$ \\
\hline
\end{tabular}

TNM, tumor, node, metastasis; CBC, complete blood count; LFTs, liver function tests; UA, urinalysis; ESR, erythrocyte sedimentation rate; CXR, chest X-ray; CT, computed tomography; MRI, magnetic resonance imaging. The value in each cell represents the number of times the practitioner requests each test or other modality. ${ }^{a} \mathrm{p}<0.05$. Significant differences exist among the cells in the column by US Census Region. ${ }^{\mathrm{b}}$ Respondents from Puerto Rico were grouped in the South Atlantic Region.

This study compared the effect of MCOs, MSAs and U.S. Census Regions on the utilization of surveillance modalities following resection of extremity STSs. Our analysis suggests that regional variations in the costs of various tests, distribution of specialists and MCO restrictions have only a modest effect on the variation in the utilization of different tests for follow-up extremity STS.

For our MSA analysis, it was assumed that physicians only treat patients from the MSA in which their office is located and that there is little cross over among MSAs. Comparison was conducted among 8 MSAs, which all provided 15 or more respondents, an 'other' MSA category consisting of all remaining MSAs with less than 15 respondents, a non-MSA representing respondents from small towns and rural areas, and lastly a 'foreign' category for respondents outside the US. Means among nine MSA categories varied minimally, indicating that surveillance patterns were not greatly dependent upon geographic factors.

MCOs have changed in many ways since their introduction in the early 1980s. The aim of managed care has been described as cutting the cost of health care while maintaining quality, yet the evidence that it has been able to achieve these aims is decidedly mixed. The current investigation shows that areas with MCO penetration rates $81.9-96.9 \%$ reported some of the highest rates for utilization of common tests. Common modalities may be used more frequently than dictated by published guidelines due to the perception that these tests are inexpensive. Therefore, inexpensive surveillance modalities were used at least as often and generally more often in areas of high penetration rates compared to those with lower penetration rates. However, the means differed little among between our four MCO penetration-rate categories for 
uncommon modalities, suggesting that expensive surveillance modalities were being used at least as often and generally more often in areas of higher MCO penetration rates than in MCOs with lower penetration rates.

The analysis by US Census Regions allows comparison by location only. The analysis showed minimal variation among the 10 Regions. This implies that surveillance strategies do not differ significantly clinically across the US. Since it is clear that the distribution of SSO members across the US is not uniform, perhaps the observed pattern is a reflection of the similarity in medical education programs across the US. It may also be due to the increasing influence of planned continuing medical education events and activities intended to improve the knowledge base of physicians in the US $(24,25)$. Rydholm provides an example of the effectiveness of continuing medical education (26). In a Swedish center guidelines were formulated, based on epidemiological data, for surgical referral of all patients with soft tissue tumors. Repeated lectures were given at local hospitals explaining the advantages of the guidelines. The same information was given to all medical students during pathology coursework and again during coursework in general surgery and orthopedics. Doctors who referred patients with STS before surgery were sent personal letters outlining the specific advantages for their patients. Over a 10 -year period, this resulted in a change in referral patterns, such that $80 \%$ of all patients in the Region with a deep-seated STS of the extremity were referred to the designated center before treatment.

The percentage of non-US respondents was $14 \%$ of the total number of respondents. Though oversampled, non-US respondents constituted only a small portion of the total respondents and were not a large enough group to analyze by country or continent. Altogether, the study revealed that there was no clinically significant variation in the utilization of surveillance modalities among MSA, MCO and US Census Regions, with the exception of office visits, urinalysis, electrolytes, site MRI, site X-ray and site CT.

Office visits and urinalyses were both statistically significantly different among MSAs and MCOs while electrolytes, site X-rays and site CTs were statistically significantly different among MSAs. The differences in surgeon practice among MSAs, MCOs and US Census Regions for the use of all surveillance modalities were all clinically small. These results imply that there is a general agreement among physicians from different MSAs, MCOs and US Census Regions regarding the use of available follow-up tests despite the lack of either a strong evidence base or consensus-based guidelines around the world. This is in agreement with studies for other tumor types. However, although there appears to be a general consensus among experts regarding the frequency of use of the common modalities, this does not hold well for uncommon modalities. The current study has obvious limitations. It was sent only to members of the Society of Surgical Oncology. Also, though extremity STSs are mainly treated via surgical procedures and surgeons tend to monitor their patients personally, some may be sent back to their referring doctors or other specialists such as medical oncologists for surveillance. This limits the generalizability of the results above. Another limitation is that the study data are based on self report. It is well known that physicians have a tendency to overreport. As well, surgeons other than SSO members operate on patients with STS.

In conclusion, our survey indicates that MSA and MCO penetration rates have only a minimal effect on the variation in the use of follow-up modalities by sarcoma surgeons. US Census Region appeared to have no effect on the utilization of surveillance modalities. Of the most commonly used tests, only the use of office visit seemed to be influenced by MSA and $\mathrm{MCO}$ penetration rates, while there was a variation in the utilization of urinalysis, electrolytes, site X-ray and site CT among surgeons practicing in different MSAs and among those practicing in areas with different MCO market share.

\section{Acknowledgements}

A portion of this material was presented at the 2007 meeting of the American Society of Clinical Oncology (J Clin Oncol 25: S627, 2007 (abs. 14533)] and at the 2008 meeting of the Association for Academic Surgery (J Surg Res 144: 292-292, 2008).

\section{References}

1. Whooley BP, Gibbs JF, Mooney MM, McGrath BE and Kraybill WG: Primary extremity sarcoma: what is the appropriate follow-up? Ann Surg Oncol 7: 2-3, 2000.

2. Singer S, Demetri GD, Baldini EH and Fletcher CD: Management of soft-tissue sarcomas: an overview and update. Lancet Oncol 1: 75-85, 2000.

3. Mann GB, Lewis JJ and Brennan MFL: Adult soft tissue sarcoma. Aust N Z J Surg 69: 336-343, 1999.

4. Alderman AK, Kim HM, Kotsis SV and Chung KC: Upperextremity sarcomas in the United States: analysis of the Surveillance, Epidemiology, and End Results database 19731998. J Hand Surg 28: 511-518, 2003.

5. Pisters PW: Combined modality treatment of extremity soft tissue sarcomas. Ann Surg Oncol 5: 464-472, 1998.

6. Lewis JJ, Leung D, Casper ES, Woodruff J, Hajdu SI and Brennan MF: Multifactorial analysis of long-term follow-up (more than five years) of primary extremity sarcoma. Arch Surg 134: 1901-1994, 1999.

7. Stojadinovic A, Jaques DP, Leung DH, Healey JH and Brennan MF: Amputation for recurrent soft tissue sarcoma of the extremity: indications and outcomes. Ann Surg Oncol 8: 509-558, 2001.

8. Brennan MF: Follow-up is valuable and effective: true, true and unrelated? Ann Surg Oncol 7: 9-14, 2000.

9. NCCN Clinical Practice Guidelines in Oncology - Soft Tissue Sarcoma, V. 2.2009 (7-15-2009, www.nccn.org).

10. Boyer KL, Ford MB, Judkins AF and Levin B (eds): Primary Care Oncology. W.B. Saunders Co., Philadelphia, PA, 1998.

11. Powell TM, Thompsen JP, Virgo KS, Johnson ET, Chan D, Colberg JW, Ornstein DK and Johnson FE: Geographic variation in patient surveillance after radical prostatectomy. Ann Surg Oncol 7: 339-345, 2000.

12. Johnson FE, Naunheim KS, Coplin MA and Virgo KS: Geographic variation in the conduct of patient surveillance after lung cancer surgery. J Clin Oncol 14: 2940-2949, 1996.

13. Johnson FE, McKirgan LW, Coplin MA, Vernava AM, Longo WE, Wade TP and Virgo KS: Geographic variation in patient surveillance after colon cancer surgery. J Clin Oncol 14: 183-187, 1996.

14. County and City Data Book 1994. U.S. Bureau of the Census. Washington, DC: The Bureau, 1995 [producer]. Albany, NY: State Data Center Program [distributor].

15. Baer LD, Johnson-Webb KD and Gesler WM: What is rural? A focus on urban influence codes. J Rural Health 13: 329-333, 1993.

16. Larson SL and Fleishman JA: Rural-urban differences in usual source of care and ambulatory service use: analyses of national data using Urban Influence Codes. Med Care 41: 65-74, 2003.

17. Ghelfi LM and Parker TS: A county-level measure of urban influence. Rural Development Perspectives 12: 32-41, 1997. 
18. Gottlieb S: US health maintenance organisations: better care from non-profit plans. BMJ 319: 211, 1999.

19. Fairfield G, Hunter DJ, Mechanic D and Rosleff F: Managed care origins, principles and evolution. BMJ 314: 1823, 1997.

20. Beitler AL, Virgo KS, Johnson FE, Gibbs JF and Kraybill WG: Current follow-up strategies after potentially curative resection of extremity sarcomas. Results: A survey of the members of the Society of Surgical Oncology. Cancer 88: 777-785, 2000.

21. Sakata K, Johnson FE, Beitler AL, Kraybill WG and Virgo KS: Extremity soft tissue sarcoma patient follow-up; tumour grade and size affect surveillance strategies after potentially curative surgery. Int J Oncol 22: 1335-1343, 2003.

22. Sakata K, Beitler AL, Gibbs JF, Kraybill WG, Virgo KS and Johnson FE: How surgeon age affects surveillance strategies for extremity soft tissue sarcoma patients after potentially curative treatment. J Surg Res 108: 227-234, 2002.
23. 2000 NRC Healthcare Market Guide (www.nationalresearch. com).

24. Davis D, O'Brien AT, Freemantle N, Wolf FM, Mazmanian P and Vaisey TA: Impact of formal continuing medical education: do conferences, workshops, rounds, and other traditional continuing education activities change physician behavior or health care outcomes? JAMA 282: 867-874, 1999.

25. Mazmanian PE and Davis DA: Continuing medical education and the physician as a learner. Guide to the evidence. JAMA 288: 1057-1060, 2002.

26. Rydholm A: Improving the management of soft tissue sarcoma. BMJ 317: 93-94, 1990 . 